\title{
A Technical Capability Evaluation Model Based Concept and Prerequisite Relation in Computer Education
}

\author{
Jiwen Luo, Tao Wang, Junsheng Chang, and Xiaoting Guo \\ National Key Laboratory for Parallel and Distributed Processing \\ College of Computer, National University of Defense Technology \\ Changsha, 410073, China \\ Email: \{luojiwen18, taowang2005, cjs7908, guoxiaoting18\}@ nudt.edu.cn
}

\begin{abstract}
Effectively assessing the results of users' online learning and enhancing social recognition has become a major development direction for online education platforms. For computer education, this article constructs a technical capability assessment model. This model integrates professional concepts in the field of computer science and extracts knowledge concepts from educational resources. The model first extracts candidate concepts, then uses a graph propagation algorithm to quantify candidate concepts and obtains concepts from them, and finally uses prerequisite relationships to further quantify the concepts mastered by students. The model combines the prerequisite relationship among concepts to quantify the skills that students have mastered. It can not only effectively evaluate the user's skill mastery but also lays a foundation for subsequent course recommendations and career recommendations for users. The model is tested in the real learning environment of 250 students. This model has been proved to own certain practicability and reliability by Kendall rank correlation coefficient, which is used as an evaluation index.
\end{abstract}

Index Terms-concept extraction; technical capability evaluation model; online learning

\section{INTRODUCTION}

In recent years, massive online open courses (MOOCs) have been developing rapidly, providing convenient education for more than 100 million users worldwide [1]. A survey from Coursera shows that MOOCs are indeed beneficial to learners who complete the course. As the survey implies, $61 \%$ and $72 \%$ of respondents reflected that MOOCs benefit education and career respectively [2]. However, with the rapid development of MOOCs, related problems and challenges have emerged as well, such as poor continuous learning, high dropout rate, lack of personalized training, insufficient practical training, and so on. Meanwhile, MOOCs platforms have been criticized for their low completion rate [3]. The average course completion rate of edX is only 5\%, and the completion rate of China's MOOCs platform-XuetangX is $4.5 \%$ [4].

To enhance the autonomy and continuity of users' learning, MOOCs platforms have made some efforts to expand their

This research was partially supported by the National Key Research and Development Program of China (No. 2018AAA0102304).

DOI reference number: 10.18293/SEKE2021-187 social recognition. The MOOCs platform launched a microdegree certification model. For example, Udacity's data analysis micro-degree certificate is completed in cooperation with Facebook and MongoDB.

Besides, online education platforms and job search websites have started cooperation. In 2015, LinkedIn, an American professional networking site, announced the acquisition of Lynda, an online teaching site founded in 1995. LinkedIn's CEO Jeff Weiner said, "The mission of LinkedIn and the mission of lynda.com are highly aligned. Both companies seek to help professionals be better at what they do. lynda.com's extensive library of premium video content helps empower people to develop the skills needed to accelerate their careers. When integrated with the hundreds of millions of members and millions of jobs on LinkedIn, lynda.com can change the way in which people connect to opportunity [5]."

With the rapid development of information technology, computer professionals are often required to possess a variety of knowledge. Paying attention to the recruitment needs of computer professionals, we find that the requirements in the recruitment notice mainly focus on technical capabilities, including mastery of programming languages, technical frameworks, related tools, related project experience, and so on. For example, different programming languages, algorithms, data structures, computer networks, technical frameworks, frontend and back-end development, etc.

For online education platforms, providing competency certification and increasing employment opportunities will be the development direction of the platform to enhance its user stickiness. For users, obtaining certification on the education platform and increasing employment opportunities will be their motivation for continuous learning.

Therefore, to increase the user stickiness of online education platforms and promote better adaptive learning of students, this article proposes a technical capability evaluation model to measure the breadth of knowledge acquired by students for computer education. This model mainly evaluates the knowledge points that students have mastered on the online learning platform. For computer-related majors, skill points are a key factor in their employment. 
The main contributions of this article are summarized as follows:

- Extract candidate concepts from online education resources.

- Evaluate the quality of candidate concepts and extract high-quality phrases to complete the concept extraction of educational resources.

- Mine the prerequisite relationships of concepts from an existing open-source knowledge base.

- Integrate the relationship between knowledge to quantify the score of each concept.

- Construct a technical ability evaluation model based on the above content.

The main innovations of this article are listed below:

- Integrate MOOCCube ${ }^{1}$ (A Large-scale Data Repository for NLP Applications in MOOCs) datasets and use graph propagation algorithms to label teaching resources in the computer education field.

- Combine the prerequisite relationship to quantify the score of concepts.

- Train a technical ability evaluation model based on the users' online learning data in a real environment.

The mark of educational resources completed in this article can be used for follow-up tracking of students' learning status, provide help to understand the mastery of specific knowledge points and serve as the basis for recommending courses and other recommended applications. On the one hand, the technical ability assessment model proposed in this paper can help users understand their knowledge. On the other hand, it can accurately recommend talents for the job market.

\section{RELATED WORK}

Related work mainly includes the following three aspects: student ability assessment in online education, curriculum concept extraction, and prerequisite relationship extraction.

Capability Assessment. In recent years, students' ability models of online education mostly measure the change of students' ability in the field of knowledge tracking [6]-[8], which evaluates the knowledge mastered by students based on the marked knowledge points. Some researchers excavate and analyze students' learning activities [4] and make summative evaluations of students' performance [9], [10]. There is also some corresponding work in the comprehensive evaluation of students that combines online learning process data and results data [11]. However, the evaluation of students' technical ability in the field of computer education remains unsolved.

Concept extraction. About concept extraction in the field of natural language processing, researches mainly comprise supervised and unsupervised methods. Supervised methods are used to train classifiers. Unsupervised methods are commonly used in TF-IDF, TextRank, and so on. Different from keyword extraction, concept extraction of MOOCs resources is faced with fewer relevant documents, short texts, and fewer words (usually only the corresponding document introduction

\footnotetext{
${ }^{1}$ http://moocdata.cn/data/MOOCCube
}

provided by teachers, which can be regarded as PPT with subtitles), especially the courses of computer major, which have the characteristics of domain knowledge extraction and are highly professional. There are few studies on keyword extraction of MOOCs resources, such as the course concept extraction [12] and course concept expansion [13].

Prerequisite relationship extraction. Inferring concept prerequisites from course dependencies or video-based course data are relatively new areas. Some of classical methods include CGL [14], CPR-Recover [15], MOOC-RF [16], and PREREQ [17].

To the best of our knowledge, our paper is the first to quantify concepts score based on prerequisite relationships after the completion of concept extraction, and apply it to student ability assessment.

\section{OUR APPROACH}

Based on the concept extraction of educational resources, we constructed a a technical ability evaluation model, which includes a candidate concept extraction module, a prerequisite relationship extraction module, a candidate concept score quantification module, and a parameter fitting module. This is shown in Figure 1.

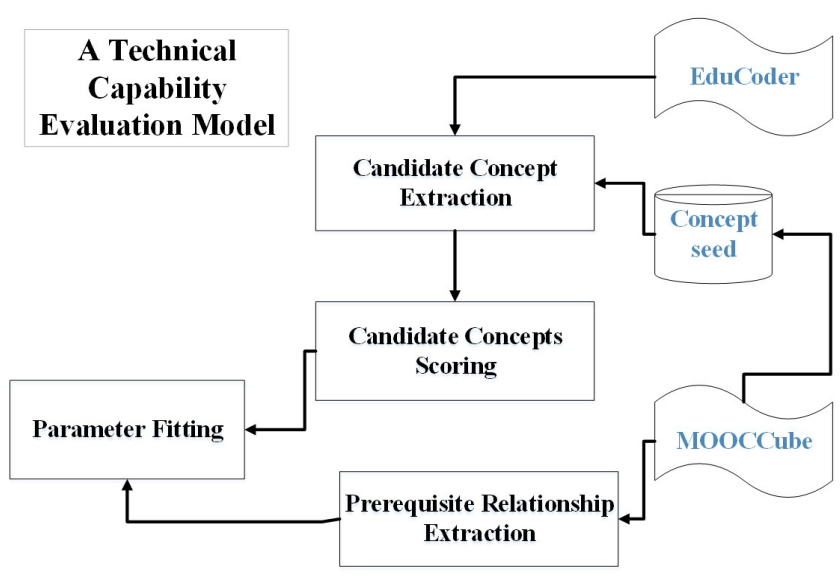

Fig. 1. Overview of our approach.

The candidate word extraction module mainly performs keyword extraction. The prerequisite relation extraction module extracts knowledge concepts with prerequisite relations from the existing knowledge base. The candidate concept scoring and quantification module use graph propagation algorithms to combine sequential knowledge concepts after constructing the knowledge graph. Perform quantitative scoring, and the parameter fitting module is result-oriented to test the impact of different functions and coefficient settings on the technical capability evaluation model.

The technical ability assessment model is designed to measure the technical ability of students. Technical ability is represented by the knowledge points mastered by students. Knowledge points are concepts acquired from educational resources. The acquisition of the concept mainly includes the 
following three steps. The first step is to extract keywords or candidate concepts from educational resources. The second step is to obtain high-quality phrases from candidate concepts. The third step is to evaluate high-quality phrases to obtain concepts. The third step depends on the human judgment after high-quality phrases were scored.

\section{A. Candidate Concept Extraction}

For computer-related majors, skill points are a key factor in their employment. The recruitment needs of Internet companies often lead to technical requirements. Therefore, we mine the skills that students may master from the courses they have completed.

We want to extract domain-specific concepts from these educational resources (such as lectures, video captions, knowledge introductions, etc.). First, we extract candidate concepts (i.e. keywords) from educational resources.

A course corpus $D$ is composed of $|D|$ courses in the same subject area.

$$
D=\left\{\text { Course }_{j}\right\}_{j=1}^{|D|}
$$

Each Course is composed of $m_{j}$ educational resources.

$$
\text { Course }_{j}=\left\{M_{i j}\right\}_{i=1, \ldots, m_{j}}
$$

Course concept $C$ can be considered as a collection of topics taught in the course. Formally, each concept in the set of course concepts $C$ can be expressed as a candidate, which is defined as a k-gram in $D$.

The problem could be formally defined as: given a set of educational data in one domain, extract domain-specific concepts $C$ from $D . C$ is a collection of concepts.

$$
C=\left\{\text { candidate }_{i}\right\}_{i=1}^{|C|}
$$

The pseudocode for candidate word extraction (i.e. extract $C$ from $D)$ is Algorithm1. We employ the linguistic pattern $((\mathrm{A} \mid \mathrm{N})+\mid(\mathrm{A} \mid \mathrm{N}) *(\mathrm{NP}) ?(\mathrm{~A} \mid \mathrm{N}) *) \mathrm{N}$ [18] to determine whether a candidate word is a noun. The isNoun() function is used in pseudocode. Candidate concept extraction mainly includes preprocessing, word segmentation, part-ofspeech tagging, judging whether it is in the vocabulary and whether it satisfies the defined linguistic pattern.

Through the above algorithm, we complete the candidate word extraction, but not all phrases extracted are domainspecific. For example, basic theory is a good phrase, but it is not a domain-specific concept. A domain-specific concept should satisfy (1) Phrasal: it is a semantically and syntactically correct phrase. (2) Informational: it is a scientific or technical concept related to the course in $D$ [12]. Therefore, we construct a weighted undirected graph and use the graphbased propagation algorithm to sort the vertices of the graph to identify high-quality candidate words.

Besides, not all phrases from candidate concepts extraction are domain-specific, e.g., the basic theory is a good phrase, but it is not a domain-specific concept. A high-quality phrase should combine the phraseness and informativeness information [19].

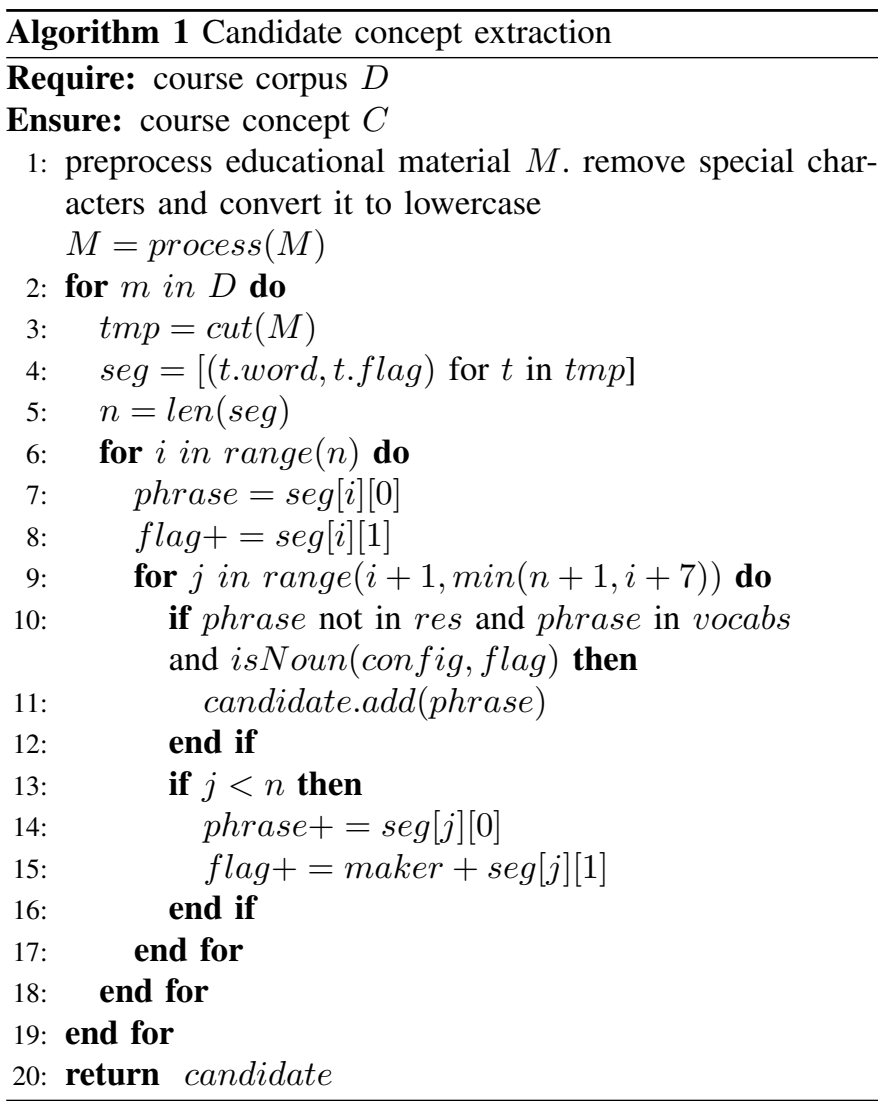

We construct a weighted undirected graph and use the graph-based propagation algorithm to sort the vertices of the graph to identify high-quality candidate words(i.e. concepts or key points) [12].

\section{B. Construction of Graph}

First, a weighted undirected graph $G=(V, E)$ was constructed, where $V$ is the vertex set of $G$ and $E$ is the edge set of $G$. Each vertex $V$ is a phrase $p_{i} \in P$ with a quality score $Q\left(p_{i}\right) . P$ is a set of high-quality phrases extracted from $D$, and $P=\left\{p_{i}\right\}_{i=1}^{|P|}$.

$$
\begin{cases}Q\left(p_{i}\right)=1, & \text { if } p_{i} \text { in concept seed } \\ Q\left(p_{i}\right)=0, & \text { if } p_{i} \text { not in concept seed }\end{cases}
$$

Concept seed is a high-quality professional vocabulary based on the text material to be processed. For each edge $\left(p_{i}, p_{j}\right) \in E$, its weight $w\left(p_{i}, p_{j}\right)$ is the semantic relatedness of the two phrases $p_{i}$ and $p_{j}$. We used a pretrained BERT [20] model to get pre-trained word contextual embeddings, and then obtained the semantic representation of each phrase via the vector addition of its word vectors. Finally, the semantic relatedness of two phrases is defined as the cosine similarity of their vectors.

\section{Graph Propagation Process}

The sorting method based on propagation was performed on a graph $G$. It is assumed that the high-confidence concept in the graph can propagate its confidence value to neighbor 
nodes with high semantic relevance to discover other potential domain-specific concepts [21]. This section contains step to obtain high-quality phrases from candidate concepts.

Each vertex $p_{i}$ has a confidence score $\operatorname{con} f\left(p_{i}\right)$ of being a domain-specific concept and $\operatorname{con} f^{k}\left(p_{i}\right)$ is the score of $p_{i}$ in the $k-t h$ iteration of the propagation.

We set the initial confidence score of each vertex as $\operatorname{con} f^{0}\left(p_{i}\right)=1$. The propagation functions are defined as:

$$
\operatorname{con} f^{k+1}\left(p_{i}\right)=1 / Z\left(\frac{\sum_{p_{j} \in A\left(p_{i}\right)} s^{k}\left(p_{j}, p_{i}\right)}{\left|A\left(p_{i}\right)\right|}\right)
$$

The voting score function as follows.

$$
s^{k}\left(p_{j}, p_{i}\right)=\operatorname{opf}\left(p_{i}, p_{j}\right) \cdot Q\left(p_{j}\right) \cdot e\left(p_{i}, p_{j}\right) \cdot \operatorname{conf}^{k}\left(p_{j}\right)
$$

$s^{k}\left(p_{i}, p_{j}\right)$ is the voting score that $p_{j}$ propagates to $p_{i}$ in the $k-t h$ iteration which is determined by opf $\left(p_{i}, p_{j}\right), Q\left(p_{j}\right)$, $e\left(p_{i}, p_{j}\right)$, and $\operatorname{conf}^{k}\left(p_{j}\right)$.

opf $\left(p_{i}, p_{j}\right)$ is the overlapping penalty between $p_{i}$ and $p_{j}$. If $p_{i}$ and $p_{j}$ contain one or more identical words, we say they are overlapping and should be penalized during the propagation process.

$$
\left\{\begin{array}{l}
\operatorname{opf}\left(p_{i}, p_{j}\right)=1, \text { if not overlapping } \\
\operatorname{opf}\left(p_{i}, p_{j}\right)=\lambda, \text { if overlapping } \lambda \in(0,1)
\end{array}\right.
$$

$Q\left(p_{j}\right)$ is the quality score of of $p_{j}, e\left(p_{i}, p_{j}\right)$ is the semantic relatedness between $p_{i}$ and $p_{j}$, and $\operatorname{con} f^{k}\left(p_{j}\right)$ is the confidence score of $p_{j}$ in the $k-t h$ iteration.

$\operatorname{con} f^{k+1}$ is the new confidence score of $p_{i}$, which is dependent on the average voting score of vertexes in $A\left(p_{i}\right)$. $A\left(p_{i}\right)$ is the vertex set that will propagate the voting scores to $p_{i}$ in each iteration. After each iteration, the confidence scores should be normalized, so $Z$ is the normalization factor.

To determine when the iteration process stops, a termination set $F$ was introduced. $A r^{k}(F)$ is the average ranking of concepts in $F$ after the $k-t h$ iteration, if $A r^{k+1}(F)>A r^{k}(F)$ the propagation process terminates.

\section{Prerequisite Relationship Extraction}

To reasonably determine the weight of concepts extracted from educational resources, we need to consider the concept relationships. For example, if a user has mastered the widthfirst search algorithm, he or she may already have mastered adjacency lists before. In other words, mastering the concept of B means that a user has mastered the concept of $\mathrm{A}$. That is to say, A is the prerequisite concept of B. Prerequisite relationship is also called pre-order relationship.

Prerequisite relationships are incorporated into the assessment model, which makes the ability scores depend on not only the number of learning courses but also the difficulty and quality of learning. We use files in the MOOCCube database to extract the prerequisite relationship in the computer science field based on a simple text matching algorithm.

\section{E. Technical Capability Evaluation Model}

The technical ability scoring model is defined as:

$$
\text { Score }=\sum_{i=1}^{n} \alpha \sum_{j=1}^{m} s c\left(\text { concept }_{i}\right)
$$

concept $_{i}$ stands for high-quality conceptual phrase(i.e. concept). $n$ represents the number of completed learning materials. $m$ represents the number of concepts possessed by a learning material, and $s c()$ is a function to quantify the concepts score.

1) The parameter $\alpha$ : $\alpha$ can be set according to the difficulty of the learning materials. (1) According to the time of completing the learning material, the outlier data can be removed, and the average value can be calculated and then normalized. (2) If the learning material has a marked difficulty coefficient, $\alpha$ can be obtained after quantifying the difficulty coefficient. The experiment in this article used the second method.

2) The function $s c()$ : Inspired by the graph propagation algorithm, concepts that are more related to other concepts should be more basic and common knowledge points. To explore the possible effects of different functions on the results, our experiment tested different functions for performance evaluation.

As for the setting of $\alpha$ and $s c()$, this article is result-oriented to make the constructed scoring model relatively reliable.

\section{EXPERIMENTS}

\section{A. Datasets}

The experiments were based on the data of EduCoder ${ }^{2}$, an actual web-based online programming teaching platform.

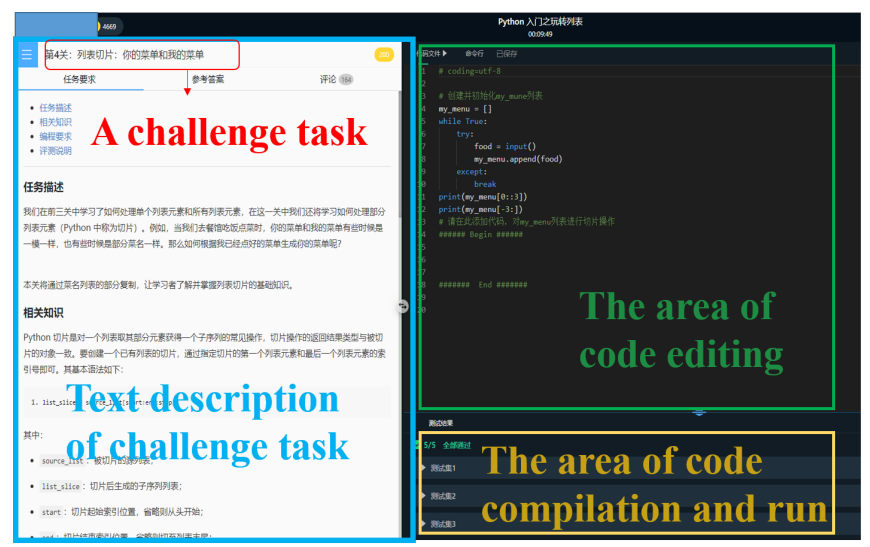

Fig. 2. A task challenge page belongs to EduCoder

In concepts extraction, 9243 challenge tasks of the platform were used for candidate concepts extraction. The text description of challenge tasks was mainly used here. In MOOCCube, 358 pairs of prerequisite relations about computer science concepts were extracted. On the EduCoder platform, the

\footnotetext{
${ }^{2}$ https://www.educoder.net/
} 
technical capability evaluation model was trained based on the data of the 250 most active users, these users have completed 50190 tasks in total.

When extracting keywords, the input text was tokenized and annotated with part-of-speech (POS) tags by jieba ${ }^{3}$. BERT is a pre-trained model in the candidate concepts ranking module, and the seed file selected 4884 computer science concepts extracted on MOOCCube.

In our experiments, a total of 548 candidate words were extracted, which were matched to the corresponding challenge task. After the candidate words were extracted, the graph propagation algorithm was used to calculate the confidence score of the candidate words, where the penalty factor $\lambda$ was set to 0.5 . We used the graph propagation algorithm in the concept sorting module to calculate the score of each concept, deleted concepts with a score of 0 , and manually deleted some concepts that were not knowledge points. Finally, we got 412 concepts.

The 412 concepts were matched with 358 pairs of prerequisite concepts extracted in MOOCCube, and 85 pairs of prerequisite concepts were identified in the concepts we extracted. Table I shows some prerequisite concepts in MOOCCube by extraction.

TABLE I

SOME PREREQUISITE CONCEPTS IN MOOCCUBE

\begin{tabular}{|c|c|}
\hline Prerequisite concept & Subsequent concept \\
\hline serial search & breadthfirst search \\
\hline array & hash function \\
\hline search & insertion sort \\
\hline computational science & dynamic allocation \\
\hline realm name & wide area network \\
\hline
\end{tabular}

Before determining the parameter $\alpha$ and the function $s c()$, the score of a concept that is in the prerequisite relationship pairs will be recalculated. If $A$ is the prerequisite concept of $\mathrm{B}$, then the score of concept $\mathrm{B}$ is the sum of the scores of all concepts in set $\mathrm{A}$, where $\mathrm{A}$ is a collection of concepts and $\mathrm{B}$ is a single concept.

\section{B. Evaluation Metrics}

Kendall's Tau [22] was used to compare the technical ability score ranking of users evaluated by this model and the actual user score ranking on the online education platform. Kendall's Tau is a non-parametric measure of relationships between columns of ranked data.

The Tau correlation coefficient returns a value of -1 to 1 . -1 means that the rank correlation of the two sequences is completely opposite, 0 means that the sequence is not related, and 1 means that the sequence is completely consistent.

$$
\text { Kendall's } \quad \text { Tau }=(C-D) / N
$$

\footnotetext{
${ }^{3}$ https://github.com/fxsjy/jieba
}

$C$ is the number of concordant pairs and $D$ is the number of discordant pairs. With $n$ denoting the number of elements in the list, $N$ is the total number of element pairs and can be calculated as below:

$$
N=1 / 2 n(n-1)
$$

\section{Experiment Results}

$\alpha$ was set to $0-10$, which corresponded to the difficulty of challenge tasks. The difficulty of tasks in the EduCoder platform was divided into five levels, which can also be regarded as the difficulty levels of educational resources.

Different function types were assigned to $s c()$, Table1 shows the calculation results of a linear function and an inverse proportional function. The value in the TableII to control sequence is Kendall's Tau obtained by comparing the similarity between the ranke calculated by skill score model and the true rank of users.

We used the equal division method to enumerate the coefficients between $[-100,100]$. The results showed that only the positive and negative of the coefficients and the choice of the function will affect Kendall's Tau value, the absolute value of the function coefficients does not affect on the result. The conclusion is consistent with Kendall's Tau's consideration of the relativity of ranking.

The paper lists four examples of positive and negative, and the coefficients of the two functions are taken as $[-2,-1,1,2]$. The data in Table II is calculated the 250 users when the prerequisite relationship between concepts were considered.

TABLE II

KENDALL'S TAU IN DIFFERENT FUNCTION

\begin{tabular}{|c|c|c|c|c|}
\hline Function coefficient & $\mathbf{- 2}$ & $\mathbf{- 1}$ & $\mathbf{1}$ & $\mathbf{2}$ \\
\hline Luction & 0.479 & 0.479 & 0.521 & 0.521 \\
\hline Linear function & 0.483 & 0.483 & 0.517 & 0.517 \\
\hline
\end{tabular}

Fixed the technical ability evaluation model Score (let $s c()$ be a linear function, and the function coefficient is 1) and got the user data of the top 50, top 100, top 150, top 200, and top 250 in EduCoder. We separately calculated Kendall's Tau value between the user ranking in the Score model and the actual user ranking without considering the prerequisite relationship. Table III shows that when the prerequisite relationship between concepts is considered, the score calculated by the score model is closer to the actual ranking.

TABLE III

KENDALL'S TAU IN DIFFERENT USER NUMBER

\begin{tabular}{|c|c|c|c|c|c|}
\hline & $\mathbf{5 0}$ & $\mathbf{1 0 0}$ & $\mathbf{1 5 0}$ & $\mathbf{2 0 0}$ & $\mathbf{2 5 0}$ \\
\hline No prerequisites relations are considered & 0.653 & 0.652 & 0.611 & 0.542 & 0.515 \\
\hline Prerequisites relations are considered & 0.647 & 0.654 & 0.614 & 0.552 & 0.521 \\
\hline
\end{tabular}

We have counted the data of the user who ranked first on the EduCoder platform. According to the technical ability evaluation model, the top ten concepts obtained by the user after learning on the platform are: data, methods, objects, 
arrays, graphs, strings, input and output, algorithm, model, and attribute.

\section{Discussion}

The effectiveness of the technology capability evaluation model mainly depends on the concepts extracted from educational resources.

This model integrates a large-scale MOOCs database and takes the prerequisite relationship between concepts into account. When Kendall's Tau is greater than 0.5, it indicates that the sorted list has a certain similarity.

By comparing various simple functions $s c()$, it can be found that the score of each concept has a low impact on the score of the user's final technical ability evaluation model. What really matters is the ranking of scores between concepts. If a better evaluation model of the users' technical abilities was expected, the order of user learning sequences and knowledge mining need to be comprehensively considered. The sequence and relevance of concepts can be used to initialize the concept graph differently to improve the graph propagation algorithm in order to better build the technical capability evaluation model.

The model has certain limitations. Our experiment believed that when a user completes a task (or learning a course) in an online education platform, the user has mastered the knowledge concept corresponding to the task (or the course) and accumulates scores for the corresponding concept. It does not take into account the time and quality of the user's completion, so the description of the user's technical ability is more like a description of the technical breadth of the user. In addition, there's still plenty of scope for improvement to distinguish whether the keywords extracted from the model belong to the knowledge concept of a specific domain.

\section{CONCLUSION AND FUTURE WORK}

To improve the social recognition of online education platforms, this paper constructed a technical ability evaluation model in the computer field based on the learning records of students on the online education platform. The technical ability evaluation model based on concepts and prerequisite relations. Its construction is mainly completed by extracting candidate concepts, calculating the importance of candidate words using graph propagation algorithm, recalculating the importance of candidate words using pre-order relations, and constructing a technical ability score model. The prerequisite relations in our research were extracted from the concept relations in the field of computer science and technology in MOOCCube.Therefore, the research conclusions of this paper are only applicable to the field of computer education. But theses research ideas can be extended to other fields.

In the future, the model is planned to be deployed on the online education platform. For online education platforms, this model can serve as a basis to provide students with ability certification, and can also provide students with jobhunting advice. A more promising direction in the future is to combine with job-hunting websites to achieve the matching of vocational skills and employment recommendations.

\section{ACKNOWLEDGMENT}

Thanks Yue Zhou and Ruolin Sun for their effective suggestions during the writing process.

\section{REFERENCES}

[1] Shah D. Year of MOOC-based degrees: A review of MOOC stats and trends in 2018 [J]. Class Central. 2019.

[2] Chen Z, Brandon A, Gayle C, et al. Who's Benefiting from MOOCs, and Why. 2015.

[3] He J, Bailey J, Rubinstein B, et al. Identifying at-risk students in massive open online courses [C]. In Proceedings of the AAAI Conference on Artificial Intelligence. 2015.

[4] Feng W, Tang J, Liu T X. Understanding dropouts in MOOCs [C]. In Proceedings of the AAAI Conference on Artificial Intelligence. 2019: 517-524.

[5] Hirsch-Allen J. The Many Reasons Why LinkedIn Bought Lynda.com. 2015.

[6] Piech C, Spencer J, Huang J, et al. Deep knowledge tracing [J]. arXiv preprint arXiv:1506.05908. 2015.

[7] Xiong X, Zhao S, Van Inwegen E G, et al. Going deeper with deep knowledge tracing. [J]. International Educational Data Mining Society. 2016.

[8] Yeung C-K. Deep-IRT: Make deep learning based knowledge tracing explainable using item response theory [J]. arXiv preprint arXiv:1904.11738. 2019.

[9] McBroom J, Yacef K, Koprinska I, et al. A data-driven method for helping teachers improve feedback in computer programming automated tutors [C]. In International Conference on Artificial Intelligence in Education. 2018: 324-337.

[10] Ruipérez-Valiente J, Muñoz-Merino P, Gascón-Pinedo J, et al. Scaling to Massiveness With ANALYSE: A Learning Analytics Tool for Open edX [J]. IEEE Transactions on Human-Machine Systems. 2017, PP (6): $1-6$.

[11] Luo J, Lu F, Wang T. A Multi-Dimensional Assessment Model and Its Application in E-learning Courses of Computer Science [C]. In Proceedings of the 21st Annual Conference on Information Technology Education. 2020: 187-193.

[12] Pan L, Wang X, Li C, et al. Course concept extraction in moocs via embedding-based graph propagation [C]. In Proceedings of the Eighth International Joint Conference on Natural Language Processing (Volume 1: Long Papers). 2017: 875-884.

[13] Yu J, Wang C, Luo G, et al. Course concept expansion in moocs with external knowledge and interactive game [J]. arXiv preprint arXiv:1909.07739. 2019.

[14] Liu H, Ma W, Yang Y, et al. Learning concept graphs from online educational data [J]. Journal of Artificial Intelligence Research. 2016, 55: 1059-1090.

[15] Liang C, Ye J, Wu Z, et al. Recovering concept prerequisite relations from university course dependencies $[\mathrm{C}]$. In Proceedings of the AAAI Conference on Artificial Intelligence. 2017.

[16] Pan L, Li C, Li J, et al. Prerequisite relation learning for concepts in moocs [C]. In Proceedings of the 55th Annual Meeting of the Association for Computational Linguistics (Volume 1: Long Papers). 2017: 1447-1456.

[17] Roy S, Madhyastha M, Lawrence S, et al. Inferring concept prerequisite relations from online educational resources [C]. In Proceedings of the AAAI Conference on Artificial Intelligence. 2019: 9589-9594.

[18] Justeson J S, Katz S M. Technical terminology: some linguistic properties and an algorithm for identification in text $[\mathrm{J}]$. Natural language engineering. 1995, 1 (1): 9-27.

[19] Shang J, Liu J, Jiang M, et al. Automated phrase mining from massive text corpora [J]. IEEE Transactions on Knowledge and Data Engineering. 2018, 30 (10): 1825-1837.

[20] Devlin J, Chang M-W, Lee K, et al. Bert: Pre-training of deep bidirectional transformers for language understanding [J]. arXiv preprint arXiv:1810.04805. 2018.

[21] Lu W, Zhou Y, Yu J, et al. Concept extraction and prerequisite relation learning from educational data $[\mathrm{C}]$. In Proceedings of the AAAI Conference on Artificial Intelligence. 2019: 9678-9685.

[22] Nelsen R. Kendall tau metric [M]. Encyclopedia of Mathematics, EMS Press, 1994. 